\title{
Electron transport in real time from first-principles
}

Uriel N. Morzan, Francisco F. Ramírez, Mariano C. González Lebrero, and Damián A. Scherlis

Citation: The Journal of Chemical Physics 146, 044110 (2017); doi: 10.1063/1.4974095

View online: http://dx.doi.org/10.1063/1.4974095

View Table of Contents: http://aip.scitation.org/toc/jcp/146/4

Published by the American Institute of Physics

\section{Articles you may be interested in}

Identification of simple reaction coordinates from complex dynamics

The Journal of Chemical Physics 146, 044109044109 (2017); 10.1063/1.4974306

Quantitative characterization of exciton from GW+Bethe-Salpeter calculation

The Journal of Chemical Physics 146, 044303044303 (2017); 10.1063/1.4974320

Communication: Improved ab initio molecular dynamics by minimally biasing with experimental data

The Journal of Chemical Physics 146, 041102041102 (2017); 10.1063/1.4974837

Perspective: On the active site model in computational catalyst screening

The Journal of Chemical Physics 146, 040901040901 (2017); 10.1063/1.4974931

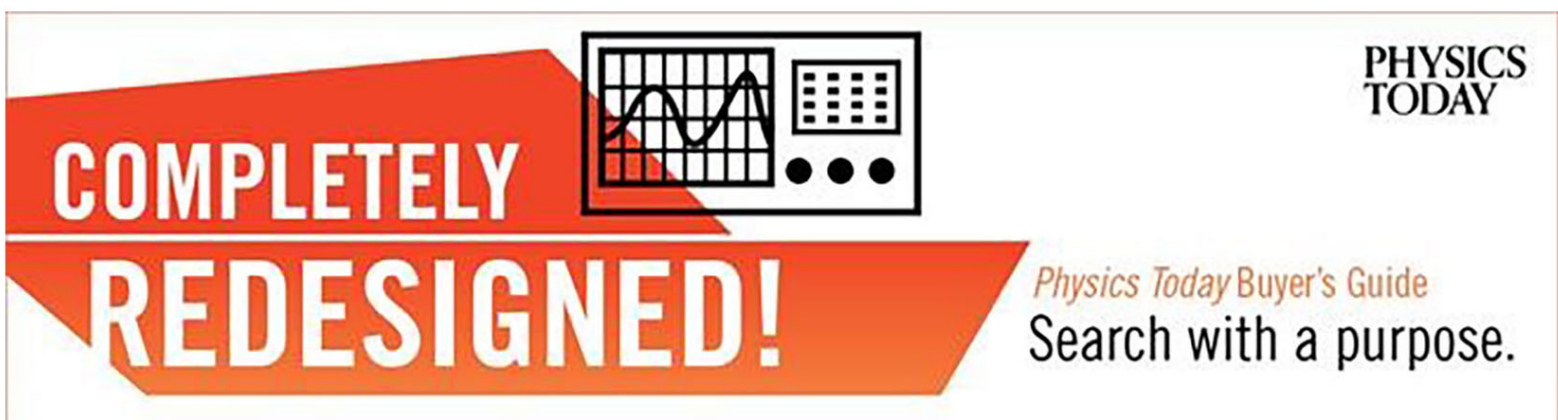




\title{
Electron transport in real time from first-principles
}

\author{
Uriel N. Morzan, ${ }^{\text {a) }}$ Francisco F. Ramírez, Mariano C. González Lebrero, \\ and Damián A. Scherlis ${ }^{a)}$ \\ Departamento de Química Inorgánica, Analítica y Química Física/INQUIMAE, Facultad de Ciencias Exactas \\ y Naturales, Universidad de Buenos Aires, Ciudad Universitaria, Pab. II, Buenos Aires C1428EHA, Argentina
}

(Received 9 April 2016; accepted 3 January 2017; published online 26 January 2017)

\begin{abstract}
While the vast majority of calculations reported on molecular conductance have been based on the static non-equilibrium Green's function formalism combined with density functional theory (DFT), in recent years a few time-dependent approaches to transport have started to emerge. Among these, the driven Liouville-von Neumann equation [C. G. Sánchez et al., J. Chem. Phys. 124, 214708 (2006)] is a simple and appealing route relying on a tunable rate parameter, which has been explored in the context of semi-empirical methods. In the present study, we adapt this formulation to a density functional theory framework and analyze its performance. In particular, it is implemented in an efficient allelectron DFT code with Gaussian basis functions, suitable for quantum-dynamics simulations of large molecular systems. At variance with the case of the tight-binding calculations reported in the literature, we find that now the initial perturbation to drive the system out of equilibrium plays a fundamental role in the stability of the electron dynamics. The equation of motion used in previous tight-binding implementations with massive electrodes has to be modified to produce a stable and unidirectional current during time propagation in time-dependent DFT simulations using much smaller leads. Moreover, we propose a procedure to get rid of the dependence of the current-voltage curves on the rate parameter. This method is employed to obtain the current-voltage characteristic of saturated and unsaturated hydrocarbons of different lengths, with very promising prospects. Published by AIP Publishing. [http://dx.doi.org/10.1063/1.4974095]
\end{abstract}

\section{INTRODUCTION}

Electron transport through molecules and nanostructures has been a field of very active research in the last decades, greatly motivated by the interest in molecular electronics and reinvigorated by the often intriguing lack of agreement between calculations and experiments. ${ }^{1-4}$ Most of the theoretical approaches currently available are based on the Landauer steady state formalism, formulated in terms of the non-equilibrium Green's function (NEGF) for coherent transport. ${ }^{5}$ In this context, a usual approximation consists in obtaining the Green's function of the system from the Kohn-Sham (KS) single-particle Hamiltonian traversed by a current. The exchange-correlation potential is approximated by the one used in time-independent density functional theory (DFT), and the charge density is calculated self-consistently in the presence of a steady state current. The effects of the leads attached to the system are represented through the corresponding self-energies. ${ }^{5-7}$ This scheme has proved useful to estimate conductance in a variety of molecules and nanoscale structures coupled to semiinfinite leads. ${ }^{8,9}$ An important limitation of these calculations is the fact that the transmission function from static DFT has resonances at the non-interacting Kohn-Sham excitation energies, which more often than not disagree with the true values.

a)Electronic addresses: umorzan@qi.fcen.uba.ar and damian@qi.fcen.uba.ar
Several developments in open or periodic boundary conditions have gone beyond the static picture. Stefanucci and other authors derived rigorous treatments within time-dependent DFT (TDDFT) for the explicit temporal evolution of the system's wavefunction or density matrix, based on the timedependent Green's function. ${ }^{10-13}$ Also within DFT, Burke, Car, and Gebauer avoided the explicit treatment of semiinfinite leads by using ring boundary conditions. ${ }^{14,15}$ All these are elegant, though computationally onerous, routes to nonequilibrium transport properties. Presently, the cost of the computations circumscribes their application to relatively simple models.

On the other hand, the microcanonical dynamics proposed by Di Ventra and Todorov, ${ }^{16}$ readdressed and implemented in a different setting by Cheng and co-workers, ${ }^{17}$ is an interesting alternative to the methodologies mentioned above. In this treatment the open-boundary conditions are substituted by a closed set of equations of motion in a finite model, where the leads must be large enough to mimic the discharge in the grandcanonical framework. The initial density for the propagation is taken from a standard DFT calculation in the presence of a bias, which is relaxed at time zero to allow the current to flow from regions of high to low potential. Di Ventra and his collaborators showed how a formally exact current between the leads is established in an "instantaneous" or quasi steady state regime. This approach removes the need to implement demanding scattering boundary conditions, but in exchange the size of the leads required to provide reasonable discharge times limits its practical use. Measurements are thus performed 
in a quasi steady state occurring in a relatively short time window before the electrons are backscattered from the boundaries of the finite leads.

Somehow in between these two general quantumdynamics frameworks - the microcanonical and the grand canonical ones-the open-boundary scheme proposed by Sanchez and co-workers is an appealing and conceptually simple method, in which the standard Liouville-von Neumann expression for the time derivative of the density matrix is augmented with a driving term. ${ }^{18}$ This term, which depends on a driving rate parameter $(\Gamma)$, allows to maintain the charge imbalance after the external potential is turned off, by restoring the elements of the density matrix associated with the leads back to the polarized state. With this strategy the backscattering inherent to microcanonical dynamics is avoided and the system can reach a steady state. Using a tight-binding model, it has been shown that this method reproduces quantitatively the result obtained with the static Landauer approach. ${ }^{18}$ Moreover, in cases where static methods yield multiple current values for a given bias, this dynamical approach is capable of selecting the most stable solution. Subotnik and co-authors have further explored the role and physical meaning of $\Gamma$, replacing the explicit representation of the leads by bath reservoirs where electrons follow an equilibrium Fermi-Dirac distribution. ${ }^{19}$ Later on, Hod and collaborators implemented a modified form of the equation of motion which led to improvements in the stability and steady-state convergence of the quantum dynamics. ${ }^{20}$ This form, which can be derived from first principles, ${ }^{24}$ was shown to conserve density matrix positivity and N-representability. In particular, they introduced a unitary transformation from the orthogonal, tight-binding atomic orbital basis to a state representation where the new basis elements can be identified with the source, drain, or device. This state representation redefines the bias voltage in terms of the coupling between the eigenstates of the isolated sections of the full system. ${ }^{20}$ Very recently, these authors generalized this approach to non-orthogonal localized basis sets using an extended Hückel model. ${ }^{21}$

Our goal in the present study is to realize a first-principles implementation of the driven Liouville-von Neumann equation discussed in the previous paragraph, suited for transport simulations in realistic molecular systems. We find that when this equation of motion, either in its original or in its modified form, is integrated in a Kohn-Sham setting with Gaussian basis functions, several issues arise which render it inapplicable to transport calculations. Namely, it does not produce a stable and unidirectional current during time propagation, and electron transport becomes strongly dependent on the $\Gamma$ value, which remains as an adjustable parameter, within certain bounds. In this article we introduce an alternative scheme that circumvents these and other difficulties observed when the driven Liouville-von Neumann approach is ported to the realm of first-principles simulations. The method is implemented in an efficient real time TDDFT code developed in our group, designed for computations in graphic processing units (GPU). ${ }^{22,23}$ The result is a powerful methodology free of adjustable parameters to accede to time-dependent electron transport properties of large molecular structures. The method is illustrated through its application to hydrocarbon polymers.

\section{DRIVEN LIOUVILLE-VON NEUMANN APPROACH}

In tight-binding implementations of the driven Liouvillevon Neumann equation, ${ }^{18,20}$ the system is divided in three regions: Source, Drain, and Molecule (S, D, and M, respectively). In this context, within an atomistic or site representation for a two lead setup, the density matrix and Hamiltonian can be written as in Equations (1) and (2), respectively,

$$
\begin{gathered}
\rho=\left[\begin{array}{ccc}
\rho_{S} & \rho_{S M} & \rho_{S D} \\
\rho_{M S} & \rho_{M} & \rho_{M D} \\
\rho_{D S} & \rho_{D M} & \rho_{D}
\end{array}\right], \\
H=\left[\begin{array}{ccc}
H_{S} & H_{S M} & H_{S D} \\
H_{M S} & H_{M} & H_{M D} \\
H_{D S} & H_{D M} & H_{D}
\end{array}\right] .
\end{gathered}
$$

The quantum-dynamics originally proposed by Sánchez and co-authors ${ }^{18}$ departs from a ground state density obtained in the presence of an electric field in the source-drain direction, which is turned off during the time propagation. In order to avoid the backscattering of the electrons at the boundaries, while keeping a voltage imbalance between the leads, open boundary conditions are introduced by augmenting the standard Liouville-von Neumman equation of motion for the density matrix with a driving term:

$$
\frac{\partial \rho}{\partial t}=-\frac{i}{\hbar}[H, \rho]-\Gamma\left(\rho-\rho^{0}\right),
$$

where $\Gamma$ is the driving rate parameter and the matrix $\rho^{0}$ can be defined as follows:

$$
\rho_{i j}^{0}=\left\{\begin{array}{l}
\rho_{i j}\left(t_{0}\right) \text { if } i, j \in S \cup D, \\
\rho_{i j}(t) \text { if } i, j \notin S \cup D .
\end{array}\right.
$$

Thus, the second term on the right hand side of Equation (3) continuously drives the charge in the leads region towards the initially polarized state but does not directly affect the evolution of the density in the central molecule region in between. For the sake of briefness, this driving term will be referred to as $\mathfrak{D}(t)$. The two contributions to $\mathfrak{D}(t),-\Gamma \rho$ and $\Gamma \rho^{0}$, can be identified with electron absorption and injection, respectively. We note that absorption and injection processes take place simultaneously, both in source and drain. It is the balance between these two contributions which defines the net amount of electrons that will be injected/absorbed in each electrode, and therefore the overall current flowing between them.

Starting from the formulation above, Hod and coworkers ${ }^{20}$ proposed a modified working expression in which the damping contribution affects not only the pure lead elements $S, D, S D$, and $D S$ but also the lead-molecule coherences $S M, M S, M D$, and $D M$,

$$
\frac{\partial \rho}{\partial t}=-i[H, \rho]-\Gamma\left[\begin{array}{ccc}
\rho_{S}-\rho_{S}^{0} & \frac{1}{2} \rho_{S M} & \rho_{S D} \\
\frac{1}{2} \rho_{M S} & 0 & \frac{1}{2} \rho_{M D} \\
\rho_{D S} & \frac{1}{2} \rho_{D M} & \rho_{D}-\rho_{D}^{0}
\end{array}\right] .
$$


It was shown in the same study that this expression can be derived from the formalism of complex absorbing potentials, in which context the addition of an imaginary potential to the Hamiltonian provokes a damping of the wavefunction and therefore a depletion of electronic density. ${ }^{20}$ The modification of the standard Liouville-von Neumann equation adding imaginary absorbing potentials of magnitude $\Gamma$ to the hamiltonian elements associated with the source and drain regions leads to a damping term as appearing in Equation (5) except for the $\rho^{0}$ elements. The final form of this equation is recovered if electron injection is represented in an analogous way, by including a potential of the same magnitude but opposite sign acting on the initial charge of the lead regions, $\rho_{S}^{0}$ and $\rho_{D}^{0}$. This ensures that the injected electrons have the equilibrium distribution of the leads subject to the external bias, assuming that deep inside the semi-infinite source and drain the electronic structure remains unperturbed. Using a tight-binding model, the authors found that expression (5) yielded an improved dynamics, preserving state occupations and density matrix positivity, accelerating the convergence to a steady state and reducing at the same time the noise in the current. ${ }^{20}$ Expression (5) has been further derived from the time-dependent non-equilibrium Green's function formalism ${ }^{24}$ and shown to be equivalent to Lindblad dynamics in the limit of infinite leads. ${ }^{25}$

\section{FIRST-PRINCIPLES FORMULATION}

The tight binding models mentioned in Sec. II use orthonormal basis sets, but many first-principles implementations as the one employed in this work are based on localized atomic orbitals. Hence, as the time propagation of the Liouville-von Neumann equation is performed in an orthonormal basis, the density matrix and hamiltonian must be transformed to an orthonormal representation to integrate the equation of motion. Throughout this article, matrices in the orthonormal basis have no specific symbol, while matrices in the non-orthogonal atomic orbitals representation are indicated with a bar (i.e., $\bar{\rho}$ and $\bar{H}$ ).

The equations of motion examined in this work were implemented in an all-electron, Gaussian basis-sets DFT code developed in our group. ${ }^{22,23}$ On the basis of GPU parallelization of the most demanding parts of the computation - which include the exchange correlation energy and the commutators between $H$ and $\rho$-and other algorithmic optimizations, this scheme can handle time-dependent simulations of molecular systems above a hundred atoms, propagated for several hundreds of femtoseconds. ${ }^{22}$ The present calculations were performed using 6-31G** basis sets in combination with the PBE exchange-correlation functional, and the equation of motion was integrated with a time step of 0.1 a.u. ${ }^{22}$

The density matrix is propagated using a Magnus expansion to first-order,

$$
\begin{aligned}
\rho(t)= & \rho(0)+\left[A_{1}(t), \rho(0)\right]+\frac{1}{2 !}\left[A_{1}(t),\left[A_{1}(t), \rho(0)\right]\right] \\
& +\frac{1}{3 !}\left[A_{1}(t),\left[A_{1}(t),\left[A_{1}(t), \rho(0)\right]\right]\right]+\cdots+\mathfrak{D}(t) \cdot(\Delta t),
\end{aligned}
$$

where $A_{1}(t) \approx \frac{1}{i \hbar} H(t / 2) t$. The total number of terms in the expansion is an adjustable parameter, typically in the order of 30 , that determines the maximum time step for which the dynamics remains stable. The value of $H(t / 2)$ in the equation above depends on the (unknown) density matrix at time $t / 2$. To deal with this, we have adopted the predictor-corrector scheme proposed by Van Voorhis, ${ }^{17}$ where $H(t / 4)$ is estimated from a linear interpolation based on the two preceding matrices, $H(-t / 2)$ and $H(-3 t / 4)$. This allows to compute $A_{1}(t / 2)$, which is used to propagate the density matrix half-step forward. The resulting $\rho(t / 2)$ is employed to construct the Kohn-Sham matrix $H(t / 2)$, with which the dynamics is evolved a full time step. Once $\rho$ has been evaluated one step forward, the KohnSham matrix needs to be updated because it depends on the density matrix via the Hartree and the exchange-correlation contributions. The Kohn-Sham matrix is assembled in the atomic or site representation; therefore, the density matrix needs to be transformed back from the orthonormal to this basis at every step, $\bar{\rho}(t)=X \rho(t) X^{\dagger}$, where $X$ is the transformation matrix that diagonalizes the overlap matrix $S$. In our implementation $X$ is computed as $U \cdot s^{-\frac{1}{2}}$, where $U$ is the matrix of eigenvectors of $S$ and $s$ the diagonal matrix containing its eigenvalues. Since the nuclei are fixed, $S$ and therefore $X$ remain constant throughout the simulation. Naturally, once the Kohn-Sham matrix is obtained, it has to be transformed to the orthonormal basis to be inserted in the Magnus expansion, $H(t)=X^{\dagger} \bar{H}(t) X$.

In order to analyze the behavior of the working formula (5) within our density functional scheme with a non-orthogonal basis set, the driving term is transformed through a canonical orthogonalization to the orthonormal representation, in which the equation of motion is propagated. The current injected or absorbed in each section of the system, $I_{X}(X=$ Source or Drain), can be computed as the contribution of $\mathfrak{D}(t)$ to the Mulliken population associated with that section,

$$
I_{X}(t)=\mathfrak{R} \sum_{i \in X, j} \overline{\mathfrak{D}}_{i j}(t) S_{j i},
$$

where $S$ represents the overlap matrix and $\mathfrak{R}$ indicates the real part. This quantity should amount to a positive number in the source, and to a negative number in the drain, which will tend to the same magnitude in the steady state. The total current flowing through the device can be defined as their average, $I(t)=\left(I_{S}(t)-I_{D}(t)\right) / 2$.

On the other hand, the off-diagonal blocks of $\overline{\mathfrak{D}}(t)$ are also responsible for a source of current different from $I(t)$ that represents a net flow directly into the molecule region. More specifically, the balance between incoming and outgoing charge contributed by the driving term in the molecule section $M$ can be similarly quantified as

$$
\Delta Q_{M}(t)=\mathfrak{R} \sum_{i \in M, j} \overline{\mathfrak{D}}_{i j}(t) S_{j i} .
$$

The index $j$ in Equations (7) and (8) spans over all the basis functions of the system, and therefore this spurious current arises from those off-diagonal elements in $\mathfrak{D}(t)$ for which $S_{i j}$ reaches high values, where $j$ is an element associated with one of the electrodes.

Figure 1 illustrates the result of the time-domain propagation of Equation (5) (panel (a) and black curve in panel (c)) 

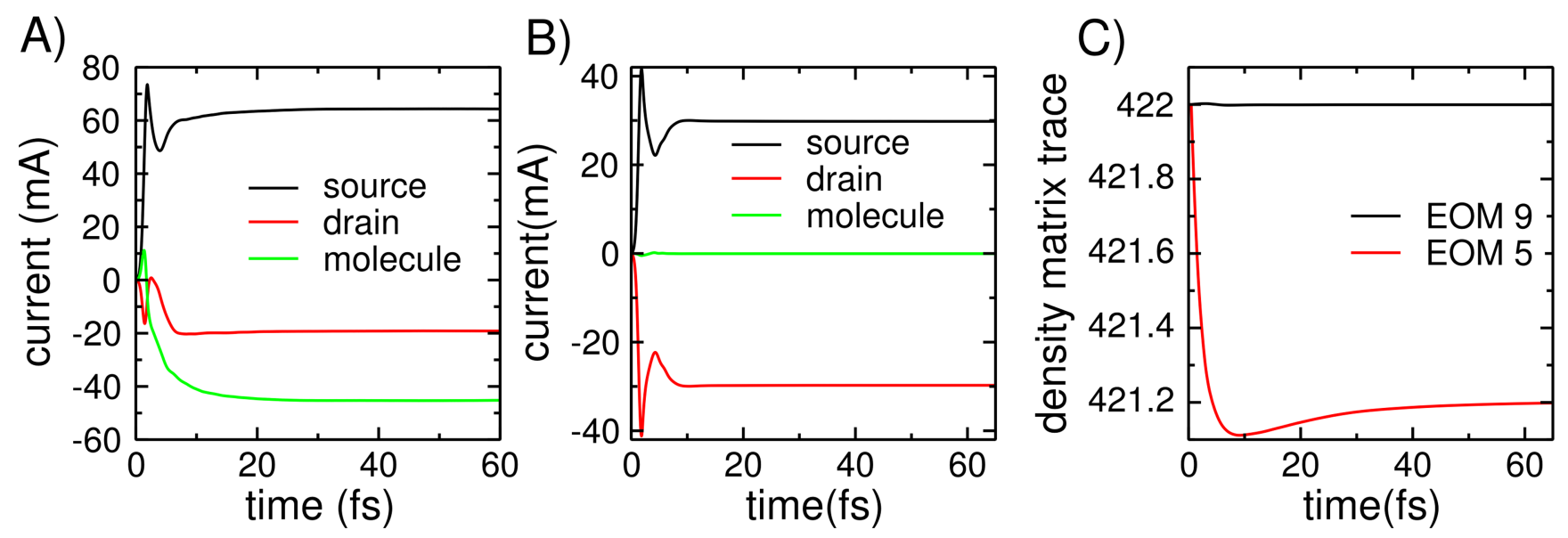

FIG. 1. Time evolution of the different components of the current $\left(I_{D}, I_{S}\right.$, and $\Delta Q_{M}$, see text) for a trans-polyacetylene chain of 60 carbon atoms, obtained from the integration of equations of motion (5) and (9) (panel (a) and (b), respectively). Panel (c) shows the evolution of the total number of electrons in the same system, as resulting from equations of motion (5) and (9).

for the case of a trans-polyacetylene chain of 60 carbon atoms $\left(\mathrm{CH}_{2}-(\mathrm{CH})_{58}-\mathrm{CH}_{2}\right)$, where the source, molecule, and drain fragments consist of 20 carbon atoms each. Panel (a) portraits an imbalance between injected and absorbed charge in the source and drain, gradually compensated by a net charge depletion occurring directly in the molecule, until a steady state is achieved. This is reflected in the red curve in panel (c), showing the total electron charge, which undergoes some oscillations and tends to stabilize after $\sim 20 \mathrm{fs}$ with a total number of particles about $0.8 e$ below the initial value. Thus, the dynamics arising from EOM (5) shows in the initial stages a slight charge depletion, leading to a steady state in which $\left|I_{S}\right| \neq\left|I_{D}\right|$, because some of the outgoing current is centered directly on the molecule, specifically, $\left|I_{S}\right|=\left|\Delta Q_{M}(t)\right|+\left|I_{D}\right|$.

Despite the treatment for injection and absorption in expression (5) is essentially identical, the non-diagonal blocks $(M S, S M, D M$, and $M D)$ in the equation of motion have only absorbing contributions. Previous applications of Equation (5) have included an "extended molecule" region. ${ }^{20,25} \mathrm{In}$ the presence of this extended region, much larger than the molecule itself, the latter is physically separated from the leads and the overlap elements coupling the electrode and the molecule sites become negligible, so that what effectively remains in the off-diagonal blocks are the mixed elements between the leads and neighboring sections of the extended molecule. This circumstance, added to the use of sufficiently large electrodes that can easily compensate for the charge drained through the off-diagonal blocks, minimizes the relative weight of the coherences in the dynamics, which in EOM (5) are damped to zero by absorbing-only contributions. In our first principles implementation, however, the incorporation of massive electrodes and a large extended molecule becomes prohibitive.

From a purely numerical point of view, the current flowing out from the molecule region can be avoided with the addition of injection in the off-diagonal elements of the driving matrix by the use of EOM (9), which enforces the coherences to remain close to their equilibrium values. This strategy eliminates incoming or outgoing currents outside the lead regions. This is visible in Figure 1, which shows that the total number of particles is well conserved with the following:

$$
\begin{aligned}
\frac{\partial \rho}{\partial t}= & -i[H, \rho]-\Gamma Y^{\dagger} \\
& \times\left[\begin{array}{ccc}
\bar{\rho}_{S}-\bar{\rho}_{S}^{0} & \frac{1}{2}\left(\bar{\rho}_{S M}-\bar{\rho}_{S M}^{0}\right) & \bar{\rho}_{S D}-\bar{\rho}_{S D}^{0} \\
\frac{1}{2}\left(\bar{\rho}_{M S}-\bar{\rho}_{M S}^{0}\right) & 0 & \frac{1}{2}\left(\bar{\rho}_{M D}-\bar{\rho}_{M D}^{0}\right) \\
\bar{\rho}_{D S}-\bar{\rho}_{D S}^{0} & \frac{1}{2}\left(\bar{\rho}_{D M}-\bar{\rho}_{D M}^{0}\right) & \bar{\rho}_{D}-\bar{\rho}_{D}^{0}
\end{array}\right] Y,
\end{aligned}
$$

where the overline denotes the atomic basis representation, and $Y=X s$.

With this equation, $\Delta Q_{\mathrm{M}}(t)$ remains practically equal to zero, while the source and drain currents reach essentially the same magnitude. In any case, in the presence of very large electrodes and extended molecule regions, the contribution of the off-diagonal terms should become less significant. As already mentioned, the index $j$ in Equation (7) spans over all the basis functions in the system, implying that large values of the overlap matrix elements $S_{i j}$ (with $i$ sitting on one of the leads) will redound to incoming and outgoing currents. With a large extended molecule, these elements of the overlap matrix will be zero for most $j$, and thus the off-diagonal elements in the driving term will have little incidence in the current computed via Equation (7). In other words, by uncoupling the bridge from the leads with the insertion of an extended molecule section, we can expect that the difference between EOM (5) and (9) eventually becomes irrelevant. In the case of systems of limited size as the ones examined here, however, damping the coherences via EOM (9) attenuates charge creation or annihilation beyond the lead regions. Expression (9) will be our master equation along this work.

The two different equations of motion discussed here can be regarded as having two distinctive natural boundary conditions. EOM (5) represents a device between electronic reservoirs at equilibrium, where the reference density matrix within the lead sections in the state representation ${ }^{20}$ should be diagonal obeying the relevant Fermi-Dirac distribution and well-defined chemical potentials. Here, since the target density is diagonal, the molecule-lead coherences are driven to 
zero during the dynamics. On the other hand, with EOM (9) an external field is used to construct the reference density matrix, resembling the state of a charged capacitor. Hence, the target density represents the equilibrium state of the entire finite junction model and not just the leads. On a purely formal view, the former boundary conditions encoding reservoirs at equilibrium provide a rigorous definition of the lead chemical potentials and electronic temperatures. Nevertheless, on a practical level the two approaches produce similar results, and the need to add non-vanishing reference terms to the leadmolecule coherences may be ascribed to the use of different boundary conditions and a finite model rather than a deficiency of EOM (5).

Figure 2 shows that increasing the lead size does not produce significant changes in the current. In other words, a polyacetylene chain of 20 carbon atoms seems to be large enough to yield converged results within the present scheme. As has been discussed in the literature, ${ }^{19,24}$ the damping term provokes an effective broadening of the density of states introducing in the leads a bulk continuum or quasi-metallic behavior (see supplementary material). Hence, the lead model does not faithfully mimic a real semi-infinite polymer, but behaves as an effective source or drain of charge. On the other hand, the particular trends illustrated in Figure 1 for equations of motion (5) and (9) are equally observed with the larger electrodes.

The current achieved in the steady-state depends on the value of $\Gamma$, as shown in Figure 3 for a fixed $\rho^{0}$. In the limit of small $\Gamma$, the standard microcanonical picture is recovered and the backscattering effect precludes any net current. The increase of the rate parameter exacerbates both injection and absorption by promoting electron exchange with the reservoirs. There is a relatively broad interval of $\Gamma$ values which maximize the conductance, above which the damping prevails and the current starts to decay. The same dependence with the rate parameter has been observed for semi-empirical hamiltonians by various authors, including Nitzan, ${ }^{19}$ Todorov, ${ }^{18}$ and to a lesser extent, by Zelovich, Kronik, and Hod in their model systems. ${ }^{20}$ While in the present DFT simulations the steady

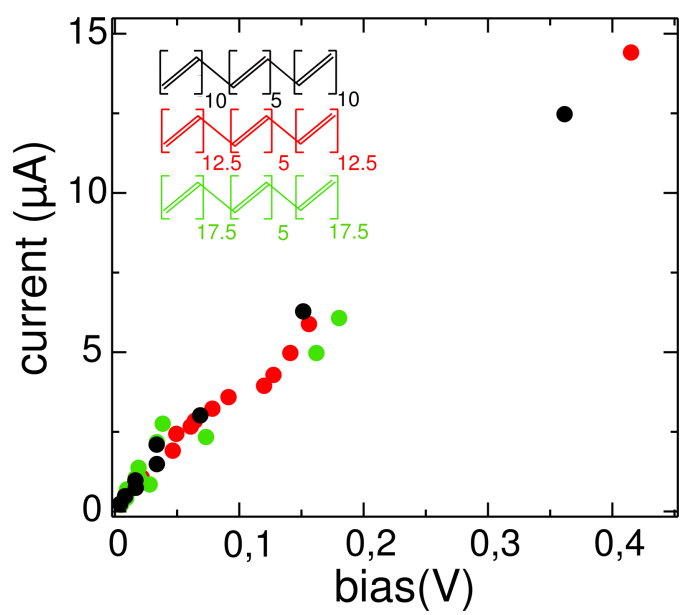

FIG. 2. Current as a function of bias for a trans-polyacetylene chain of 10 carbon atoms, using polyacetylene electrodes of various lengths. The bias has been obtained according to the dynamical approximation.

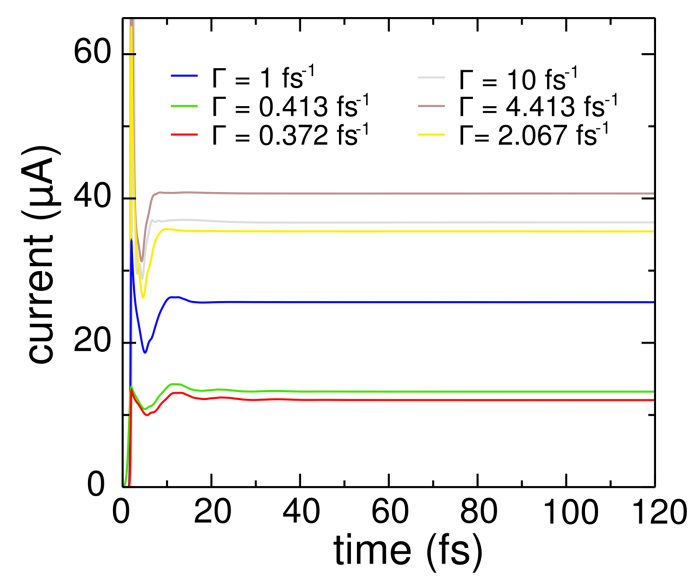

FIG. 3. Current as a function of time on a trans-polyacetylene chain of 60 carbon atoms, for different values of the rate parameter $\Gamma$ and an applied bias of $2.1 \mathrm{~V}$.

state current appears to be more sensitive to the rate parameter in comparison to previous tight-binding reports, this dependence is in any case a weakness of the driven Liouville-von Neumann approach. It has been argued that the magnitude of the driving rate should be somewhere between that of the energy spacing separating the lead levels and the effective molecule-lead coupling. ${ }^{19,24}$ Nevertheless, and despite the insight provided in the literature about the role of the driving rate parameter, ${ }^{18,20,24,26}$ a rigorous way to determine it univocally has not been proposed as yet. We will come back to this issue in Section V, where we discuss a path to obtain the current as a function of the voltage bias, which gets rid of the $\Gamma$ dependence.

\section{PERTURBING THE SYSTEM OUT OF EQUILIBRIUM}

In these simulations, the density $\rho^{0}$ involved in the construction of the driving operator is obtained from a groundstate self-consistent calculation in the presence of an applied bias. The initial density $\rho\left(t_{0}\right)$, instead, and at variance with the practice adopted in Ref. 18, corresponds to the ground-state in the absence of any external field, by which the Hamiltonian commutes with the density matrix at the initial steps of the dynamics. The upper panel of Figure 4 displays the time evolution of the Mulliken charges in the source and drain regions for a simulation performed according to this procedure in the polyacetylene molecule. Conversely to the expected behavior, neither the populations reach stable values nor the charge of the source remains above that of the drain, but they exhibit an oscillatory and overlapping evolution within this simulation time-scale.

The behavior changes dramatically if the effect of the driving term is incorporated smoothly, by multiplying the driving rate parameter times a time-dependent factor which increases gradually from 0 to 1 . In particular, the lower panel of Figure 4 shows a more meaningful behavior when the value of $\Gamma$ is controlled in the following form:

$$
\Gamma= \begin{cases}\Gamma_{0} \cdot e^{-(t-b)^{2} / c} & \text { if } t \leq b, \\ \Gamma_{0} & \text { if } t>b,\end{cases}
$$



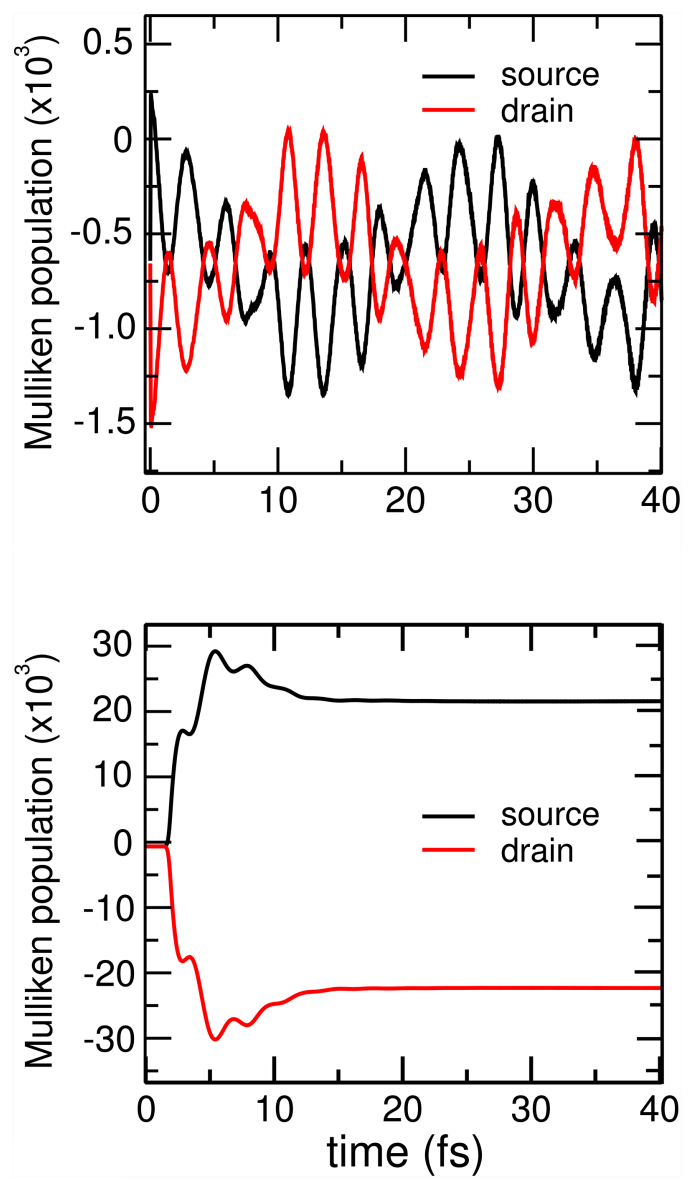

FIG. 4. Time evolution of the Mulliken charges in the source and drain regions, for a driven dynamics in a polyacetylene chain of 60 carbon atoms. The upper panel depicts the behavior when the driving term is switched on at time zero. The lower panel corresponds to a smooth inclusion of the driving term according to Equation (10).

with the chosen numerical parameters $b=2.419 \mathrm{fs}$ and $c=0.585 \mathrm{fs}^{2}$. It was checked that the specific values of $b$ and $c$ have practically no effect on the final charges and currents in the steady-state, providing that the time-scale of the perturbation is well below the one associated with the wave-packet reflection. Now, the populations of the leads reach a steadystate in which the charge difference between source and drain has the expected sign.

To rationalize these results, it must be recalled that the electron dynamics evolves according to Equation (9) in the absence of an electric field. Since the starting density corresponds to the ground-state of $H$, the value of the commutator $[H, \rho]$ is initially zero. However, the incorporation of the driving term may produce abrupt variations of the density at the initial stages of the time-propagation, when $\rho-\rho^{0}$ can be large. The irruption of the perturbation seemingly excites the accessible resonances of the electron structure, resembling the application of a step or delta-function potential in a quantumdynamics. Thus, the resulting pattern reflects the characteristic frequencies of the system, rather than the transport process itself.

The perturbing effect of the driving term could be minimized if the initial density were set equal to $\rho^{0}$. In such a case $H$ and $\rho$ would not commute and similar excitations would develop, but this could be addressed by gradually disabling the applied field in the Hamiltonian. In our scheme we choose the alternative route and use as starting density a solution of $H$. A gradual perturbation is easy to implement as a smoothed time-dependent driving rate parameter. Noteworthy, previous TDDFT studies of molecular conductance using microcanonical dynamics have reported "transient fluctuations" or "noise" in current and charges, which origin could not be established with certainty. ${ }^{17,27}$ The results presented above suggest that the fluctuating nature of those observables might have been a consequence of the sudden incorporation or removal of the bias potential in the time dependent Hamiltonian at time zero. Hence, whereas the initial magnitude of the perturbation does not seem to be an issue for tight-binding models, it appears to be a crucial ingredient in real-time conductance simulations from first-principles.

It has to be recalled that in tight-binding studies, both the system sizes and simulation time-scales are considerably larger than the ones reported here. Therefore, it is possible that for sufficiently large lead models and long enough simulation times, the effects associated with the initial perturbation observed in the present investigation could be fully damped. In this sense, the smoothing of the step-like driving term can be regarded as a strategy to accelerate the reach of the steady state in all-electron TDDFT calculations.

\section{THE BIAS POTENTIAL: DYNAMICAL APPROXIMATION}

Up to this point, the bias potential or voltage bias $(V)$ has not been given explicitly, but has remained implicit in $\rho^{0}$, which is the ground-state density in the presence of a uniform electric field of magnitude $V / d$, with $d$ the separation between source and drain. Equation (9) continuously drives the charge in the leads towards the reference density of the system equilibrated with the electric field turned on. Nevertheless, in our simulations the electronic density in the leads, $\rho(t)$, never becomes equal to $\rho^{0}$ - the difference between the two strongly depends on $\Gamma$ - and therefore it would be inaccurate to assume that the bias that led to $\rho^{0}$ in a static calculation is the same as the one developed in dynamical conditions with a driving operator formed with $\rho^{0}$. Hereafter, we will refer to these two magnitudes as the static $\left(V_{s}\right)$ and dynamical $\left(V_{d}\right)$ bias. The former is an artifact to generate $\rho^{0}$ and construct the driving term, while the latter is the effective or physical electric potential difference arising between source and drain during the electron dynamics, and is the relevant parameter to characterize conductance. Its value, however, is not known $a$ priori.

An estimation of $V_{d}$ can be accomplished in terms of the charge populations of the leads in operating conditions. In particular, it is possible to establish a link between $V_{d}$ and $V_{s}$ through a sort of calibration curve based on the charge difference between the leads. To illustrate this, Figure 5 presents on the left panel the steady state currents as a function of $V_{s}$, for various values of $\Gamma$. It can be seen that, at least in the explored range, the current increases with $V_{s}$ (due to the rise in $\rho^{0}$ ), but the $I-V$ curve is not univocally determined because, as already discussed in Section III, the conductance exhibits 

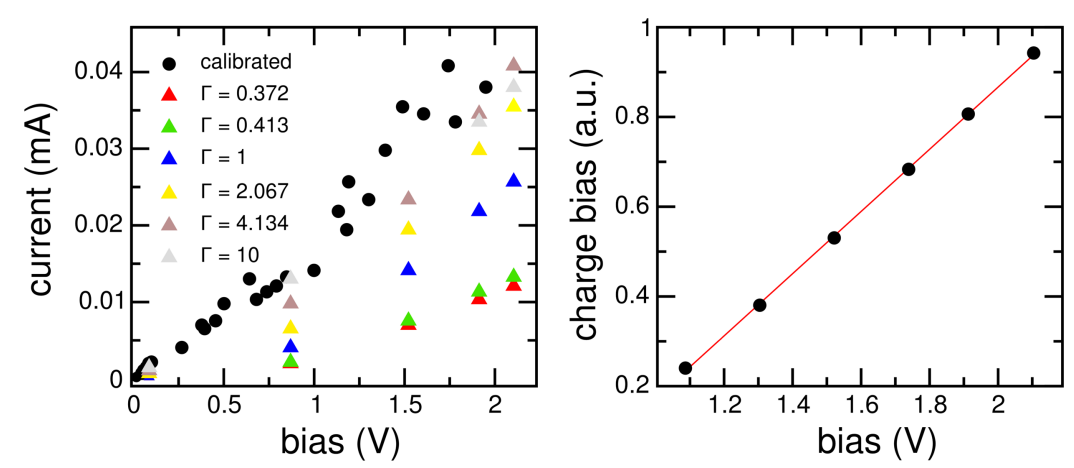

FIG. 5. Steady-state currents in polyacetylene and the calibration strategy. On the left panel, the triangles show the steady-state currents as a function of the statically applied bias $\left(V_{S}\right)$ for different values of the rate parameter $\Gamma$, in atomic units. The black circles correspond to the same data, expressed as a function of the dynamical bias $\left(V_{d}\right)$ which is assigned according to the charge difference between source and drain in the steady-state. Specifically, $V_{d}$ is interpolated from the calibration curve shown on the right panel, which collects the Mulliken charge difference surging between source and drain in a ground state calculation as a function of the applied bias. a significant dependence on the driving rate parameter. On the right panel, the Mulliken charge difference obtained in a static calculation subject to a uniform field of magnitude $V_{s} / d$ is plotted against the corresponding voltage bias $V_{s}$. This plot serves as a calibration curve from which, for any given charge difference measured in operation conditions, and in particular in the steady-state, it is possible to interpolate a bias. In this way, the effective or dynamical bias $V_{d}$ is estimated as the one that in static conditions produces the same charge population difference as in the steady-state. The black circles on the left panel of Figure 5 correspond to the same data-points obtained for all different $\Gamma$, after reassigning the bias according to the calibration curve. With this approach the dependence of the steady state current on the driving rate parameter is practically eliminated, providing a criterion to define the voltage unambiguously. The calibration also yields the alignment of the data-points on a single line for the rest of the molecules examined in this work, as it is shown in Sec. VI. Thus, by selecting the charge difference as the reference variable, this procedure offers a way to estimate the electric potential difference in the dynamical regime, reducing at the same time the ambiguity associated with the choice of the $\Gamma$ parameter.

The absolute values of electron charges or populations are irrelevant in our analysis, which consider only their variations. In particular, the charges are adopted to establish a connection between the calculated population and the bias. In this context, the role of the population scheme is just to sense the change in the potential difference, which makes the method quite robust with respect to the chosen population analysis flavor. In the supplementary material it is shown that the I-V characteristics resulting from the dynamical bias approximation calibrated with either Mulliken or Löwdin definitions do not present any significant differences.

As discussed in Section III, two bounds for the driving rate parameter have been identified in the literature: it should be larger than the energy difference between lead states, and smaller than the effective molecule-lead coupling. ${ }^{19,24}$ In a tight-binding framework with orthogonal basis sets, these two limits are well defined because the lead and molecule states are mutually orthogonal, and the energy difference between states is homogeneous. In our simulations, however, the coupling between the non-orthogonal states of the leads and the molecule cannot be unambiguously defined, and the spacing between states in the polyacetylene lead model is highly heterogeneous. For these reasons, to find the physically consistent bounds for $\Gamma$ within our scheme is not straightforward and would require additional assumptions. In any case, if $\Gamma$ is too high, the target density is enforced in the lead sections too tightly to allow for an appropriate response and relaxation at the interface, the dynamics becomes unstable, and eventually diverges. On the other hand, when the rate parameter is small, the relaxation process in the leads is not sufficiently fast to allow for the electronic density in those sections to reach $\rho^{0}$. This circumstance, however, has a lesser impact on the I-V curves obtained from the dynamical bias scheme, because by using the time dependent lead populations to estimate the voltage, this approximation can be used independently of whether the density in the leads meets the target $\rho^{0}$. In practice, the consequence of employing small values of $\Gamma$ is to limit the voltages that can be achieved within the dynamical bias approximation framework. If $\Gamma$ is far too low, then the microcanonical picture is recovered and the current is damped by the backscattering. The decrease in the current at low $\Gamma$ observed in Figure 3 originates from the low dynamical bias produced.

The calibrated $I-V$ curve tends to be coincident with the curves corresponding to rate parameters which optimize the conductance (e.g., $\Gamma=4.134 \mathrm{fs}^{-1}$ or $\Gamma=10 \mathrm{fs}^{-1}$ ). This means that with the use of these parameters, the voltage bias applied statically to polarize the system is approximately maintained during the transport process, or that the charge density developed in the steady state at the electrodes is very close to the target $\rho^{0}$. Moreover, this is in line with the analysis in Refs. 20 and 24, where it is shown that the driven Liouvillevon Neumann equation reproduces the Landauer results for those values of $\Gamma$ maximizing the currents.

At this point, it must be noticed that some degree of ambiguity may affect the definition of the static bias calculated from the applied electric field as $V_{s}=|\vec{E}| \cdot d$. Specifically, if the charge is not uniformly distributed along the leads, the voltage drop may not be homogeneous, causing a deviation from the linear relation between $V_{s}$ and $d$. This effect, in any case, should be very minor, as suggested by the $I-V$ curves and the tunneling decay constant discussed in Sec. VI.

At variance with the state representation scheme introduced in Ref. 20, the present approach lacks the possibility to directly define the target chemical potentials at the electrodes. It must be noticed, however, that the state representation transformation would not be straightforward to implement in a first-principles framework, given the fact that it requires the diagonalization of every fragment of the system considered in isolation. In a tight-binding model with appropriate 
interaction parameters, the electronic structure and matrix elements of the isolated parts will not differ too much when embedded in the whole structure. In a DFT description, instead, the molecule taken apart from the leads becomes a radical species with chemical and electronic properties substantially different from the closed-shell compound. Unless large metallic leads are employed, the same will be true for the electrodes, in particular if these are of moderate size. Therefore, the eigenvalues obtained for the isolated fragments would not be representative of the system operating as a whole.

It is interesting to note that, in the context of the LandauerBüttiker picture ${ }^{28}$ the strategy utilized in NEGF-DFT calculations or even in previous driven Liouville-von Neumann models, based on the state representation transformation, resembles the non-invasive 2-probe conductance experiment, since the voltage drop is tied to the chemical potential difference between the reservoirs, which is a fixed parameter in the simulations. On the other hand, the dynamical bias approximation proposed in our work is more akin to the 4-probe model in which the voltage bias is being measured at the limits of the molecular bridge and not between the reservoirs. In other words, the bias is not fixed but arises in the leads as a consequence of the dynamics.

\section{APPLICATION TO ORGANIC POLYMERS}

Figure 6(a) compares the current-voltage characteristic of three hydrocarbons: the polyacetylene structure with a bridge of 20 carbon atoms examined in Secs. IV and V, the same molecule with a shorter bridge of 10 carbon atoms, and a linear saturated alkane of 60 carbon atoms $\left(\mathrm{CH}_{3}-\left(\mathrm{CH}_{2}\right)_{58}-\mathrm{CH}_{3}\right)$ with $20 \mathrm{CH}_{2}$ units in the bridge. In every case, the calibration procedure based on the Mulliken charges difference between source and drain makes all data-points obtained with different $\Gamma$ values, to collapse on a single curve. The currents for the saturated hydrocarbon are between one and two orders of magnitude below those computed for the unsaturated molecule of the same size. The conductance obtained for polyacetylene increases by a factor of 3 when the length of the bridge is reduced from 20 to 10 carbon atoms. This result reflects a tunneling decay constant $(\beta)$ of $0.058 \AA^{-1}$, in good agreement with the available estimates. ${ }^{29}$ Figure $6(\mathrm{~b})$ shows that, at least for the three different polyacetylene lengths explored in this work, the method reproduces a perfectly exponential decay.

Panel (c) confronts, for a polyacetylene bridge of 14 carbon atoms, the currents obtained from our time-dependent simulations, with those calculated through the NEGF formalism using the TranSIESTA method. ${ }^{30}$ In the later calculations, the polyacetlylene chain is attached to two gold electrodes via sulfur atoms, while in the real time simulations, the leads and the bridge have the same structure. Noteworthy, and despite the distinct molecular junctions, the currents are in very good accord. As already discussed, the driving term effects a broadening of the density of states at the leads, allowing for an efficient exchange of charge. Thus, it seems appropriate to compare our results with NEGF calculations using metallic -rather than alkene-leads. As a matter of fact, the use of polyacetylene leads in NEGF simulations produces less robust results, in the sense that they show an unphysical sensitivity
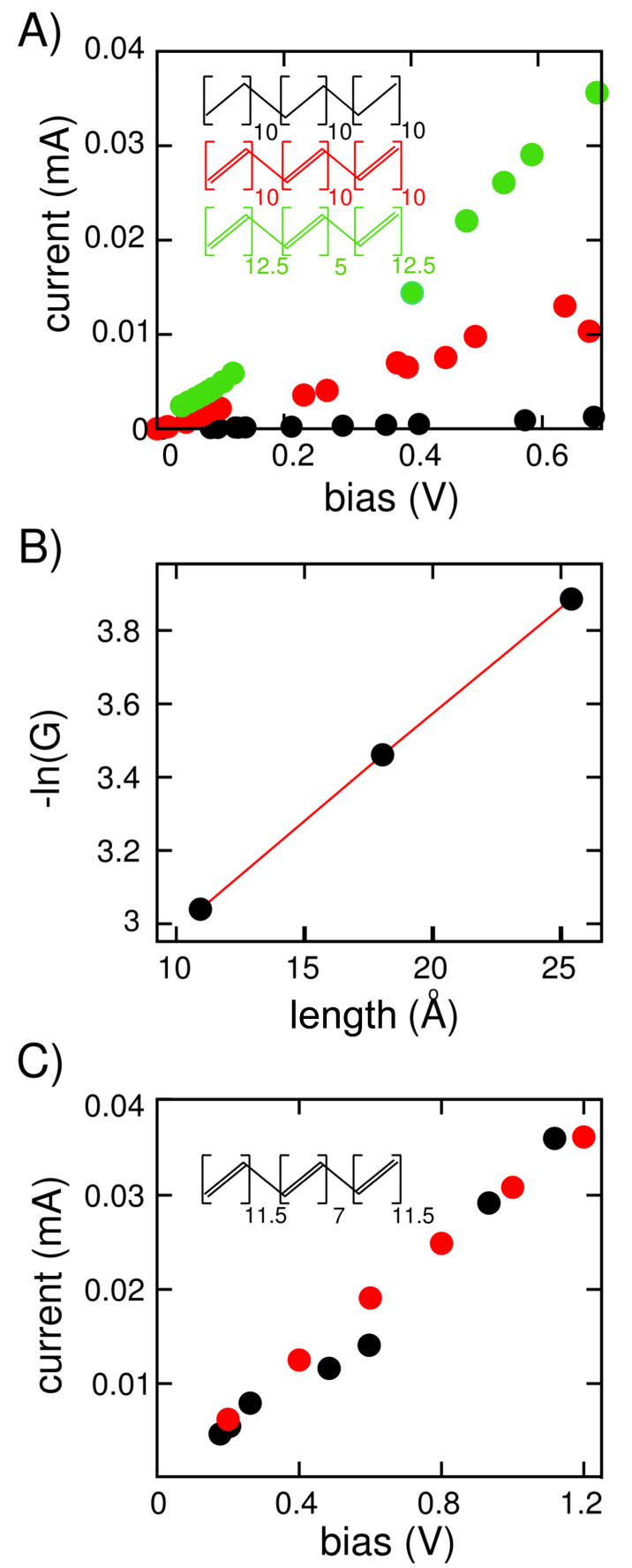

FIG. 6. $I-V$ curves computed with the driven Liouville-von Neumann approach for different organic structures: (a) a trans-polyacetylene chain with a bridge of 10 and 20 carbon atoms (green and red dots, respectively), and a saturated alkane of 60 carbon atoms with $20 \mathrm{CH}_{2}$ units in the bridge (black dots). (b) Logarithm of the conductance obtained from the current-voltage characteristics, versus the distance between leads, for the trans-polyacetylene model with bridges of 20,14 , and 10 carbon atoms. (c) $I-V$ curves for a transpolyacetylene chain of 14 carbon atoms. The red dots show results from NEGF calculations for a model connected on each end to gold electrodes through a sulfur atom (data extracted from Ref. 30). The black dots depict the steadystate currents computed through the driven Liouville-von Neumann approach, using a source and a drain of 23 carbon atoms each.

to marginal changes in bias and geometry. Transport calculations based on Green functions require electrodes exhibiting smooth and continuous density of states, conditions which are not strictly satisfied by the alkene chains, affecting the reliability of the outcome. 


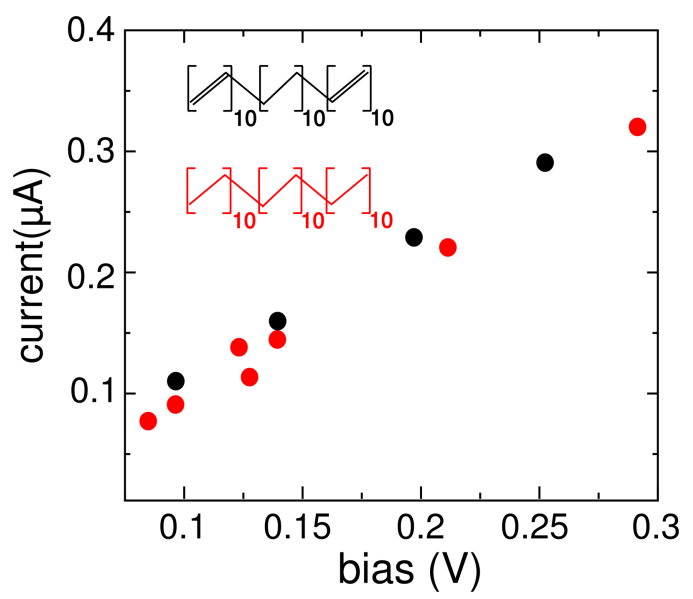

FIG. 7. $I-V$ curves for an alkane polymer of 14 carbon atoms, inserted in between leads consisting of polyacetylene (black dots) or alkane (red dots) chains.

The agreement manifested in Figure 6(c) suggests that, for these systems, the structure of the electrodes plays a minor role in the steady-state currents, as expected for weakly conducting devices as those examined in this work. In fact, the replacement of unsaturated by saturated electrodes has only a marginal impact on the overall behavior. This is depicted in Figure 7, which compares the conductance of the alkane in between polyacetylene leads, with the conductance obtained for the same polymer using alkane leads.

\section{SUMMARY}

This study presents the first the implementation of the driven Liouville-von Neumann approach for time-dependent transport in an ab initio DFT setting. The main modifications or innovations with respect to previous semi-empirical schemes are (i) incorporation of charge injection in the off-diagonal elements of the driving operator, (ii) modulation of the perturbation associated with the driving term at time zero, thus avoiding the unphysical excitation of resonances unrelated to the transport process, and (iii) adoption of the charge difference between leads as the reference variable to establish the voltage bias, which removes the dependence on the driving rate parameter.

In transport simulations based on NEGF or on semiempirical methods with large models, the chemical potential is fixed at the reservoirs, which are coupled to the molecule through leads that deviate from equilibrium at the interface. This is also the behavior of the leads in our simulations. Despite the fact that those sections are being driven to equilibrium, the density in the proximity of the interface deviates from the target distribution. In these simulations the electrodes are not supposed to represent the infinite reservoirs, which are implicitly introduced through the driving term. The addition of an extended molecule section is a good strategy to avoid the complications regarding the molecule-lead interface. Nonetheless, a small model can still provide the correct physics if the leads are driven close to, rather than exactly to, the target density. In these finite models, large reservoirs with homogeneous and well defined chemical potentials are absent, yet their role is enacted by the driving term.
The method presented in this article has proved to be an efficient and stable scheme, suitable to perform real-time electron transport simulations on systems above a hundred atoms for several hundreds of femtoseconds. In the molecules explored here, steady states were typically achieved within the first ten or twenty femtoseconds. This methodology opens the door to simulations of charge transport in realistic chemical structures, from conducting polymers to metallic nanowires to biological macromolecules. Aside from the most conventional phenomena, this method allows to explore a multiplicity of challenging and sophisticated conductance experiments, for example, time-resolved transport modulated by varying electric fields or laser pulses. In particular, although not discussed in the present work, this code offers the possibility to represent large environments through a quantummechanics molecular-mechanics approach. ${ }^{22}$ This can be useful to model the effect of a solvent or other complex media in transport processes, which will be the subject of future work.

\section{SUPPLEMENTARY MATERIAL}

See supplementary material for density of states of the polyacetylene molecule and currents obtained from the dynamical bias approach based on Mulliken and Löwdin populations.

\section{ACKNOWLEDGMENTS}

The present work is dedicated to the memory of Jorge Luis Morzan.

We thank Cristian Sanchez, Dario Estrin, and Daniel Murgida for helpful discussions. We express our gratitude to Ivan Girotto and the ICTP for technical support and GPU computing time which made possible most of the simulations presented in this study. Finally, we are thankful to the referees for their authoritative review and fundamental suggestions. This work has been funded by grants of the Agencia Nacional de Promocion Cientifica y Tecnologica de Argentina, No. PICT 2012-2292, and of the University of Buenos Aires, UBACYT No. 20020120100333BA. U.M. and F.R. acknowledge CONICET for doctoral fellowships.

\footnotetext{
${ }^{1}$ A. Nitzan and M. A. Ratner, Science 300, 1384 (2003).

${ }^{2}$ M. Di Ventra, S. T. Pantelides, and N. D. Lang, Phys. Rev. Lett. 84, 979 (2000).

${ }^{3}$ C. J. Lambert, Chem. Soc. Rev. 44, 875 (2015).

${ }^{4}$ K. H. Khoo, Y. Chen, S. Li, and S. Y. Quek, Phys. Chem. Chem. Phys. 17, 77 (2015)

${ }^{5}$ S. Datta, Electronic Transport in Mesoscopic Systems (University Press: Cambridge, United Kingdom, 1995).

${ }^{6}$ C. Yam, X. Zheng, G. Chen, Y. Wang, T. Frauenheim, and T. A. Niehaus, Phys. Rev. B 83, 245448 (2011)

${ }^{7}$ M. Brandbyge, J.-L. Mozos, P. Ordejón, J. Taylor, and K. Stokbro, Phys. Rev. B 65, 165401 (2002)

${ }^{8}$ C. Joachim and M. A. Ratner, Proc. Natl. Acad. Sci. U. S. A. 102, 8801 (2005).

${ }^{9}$ G. C. Solomon, D. Q. Andrews, R. P. V. Duyne, and M. A. Ratner, J. Am. Chem. Soc. 130, 7788 (2008).

${ }^{10}$ G. Stefanucci and C.-O. Almbladh, Europhys. Lett. 67, 14 (2004).

${ }^{11}$ G. Stefanucci and C.-O. Almbladh, Phys. Rev. B 69, 195318 (2004).

${ }^{12}$ X. Zheng, F. Wang, C. Y. Yam, Y. Mo, and G. Chen, Phys. Rev. B 75, 195127 (2007).
} 
${ }^{13}$ S. Kurth, G. Stefanucci, C.-O. Almbladh, A. Rubio, and E. K. U. Gross, Phys. Rev. B 72, 035308 (2005).

${ }^{14}$ R. Gebauer, S. Piccinin, and R. Car, ChemPhysChem 6, 1727 (2005).

${ }^{15}$ K. Burke, R. Car, and R. Gebauer, Phys. Rev. Lett. 94, 146803 (2005).

${ }^{16}$ M. D. Ventra and T. N. Todorov, J. Phys.: Condens. Matter 16, 8025 (2004).

${ }^{17}$ C.-L. Cheng, J. S. Evans, and T. Van Voorhis, Phys. Rev. B 74, 155112 (2006).

${ }^{18}$ C. G. Sánchez, M. Stamenova, S. Sanvito, D. R. Bowler, A. P. Horsfield, and T. N. Todorov, J. Chem. Phys. 124, 214708 (2006).

${ }^{19}$ J. E. Subotnik, T. Hansen, M. A. Ratner, and A. Nitzan, J. Chem. Phys. 130, 144105 (2009).

${ }^{20}$ T. Zelovich, L. Kronik, and O. Hod, J. Chem. Theory Comput. 10, 2927 (2014).

${ }^{21}$ T. Zelovich, L. Kronik, and O. Hod, J. Phys. Chem. C 120, 15052 (2016).
${ }^{22}$ U. N. Morzan, F. F. Ramírez, M. B. Oviedo, C. G. Sánchez, D. A. Scherlis, and M. C. Gonzalez Lebrero, J. Chem. Phys. 140, 164105 (2014).

${ }^{23}$ M. A. Nitsche, M. Ferreria, E. E. Mocskos, and M. C. Gonzalez-Lebrero, J. Chem. Theory Comput. 10, 959 (2014).

${ }^{24}$ L. Chen, T. Hansen, and I. Franco, J. Phys. Chem. C 118, 20009 (2014).

${ }^{25}$ O. Hod, C. A. Rodrguez-Rosario, T. Zelovich, and T. Frauenheim, J. Phys. Chem. A 120, 3278 (2016).

${ }^{26}$ T. Zelovich, L. Kronik, and O. Hod, J. Chem. Theory Comput. 11, 4861 (2015).

${ }^{27}$ J. S. Evans and T. V. Voorhis, Nano Lett. 9, 2671 (2009).

${ }^{28}$ R. Landauer, J. Phys.: Condens. Matter 1, 8099 (1989).

${ }^{29}$ H. Liu, N. Wang, J. Zhao, Y. Guo, X. Yin, F. Y. C. Boey, and H. Zhang, ChemPhysChem 9, 1416 (2008).

${ }^{30}$ J. Yao, Y. Li, Z. Zou, H. Wang, and Y. Shen, Superlattices Microstruct. 51, 396 (2012). 